\title{
Reflection seismic waveform tomography
}

\author{
Yanghua Wang ${ }^{1}$ and Ying Rao ${ }^{1}$ \\ Received 7 July 2008; revised 31 December 2008; accepted 14 January 2009; published 13 March 2009.
}

[1] In seismic waveform tomography, if using reflection data with limited source-receiver offsets, it is difficult to reconstruct the deep part of the subsurface velocity model. We present two approaches to tackle this problem: layer stripping and weighted updating. In a layer-stripping procedure, we replace the top portion of seismic data with synthetics generated from the previous-layer inversion and make the current inversion focus on the minimization of the data misfit corresponding to the deep part of the model. To improve efficiency, we use only sparsely sampled frequency data in the deeper-layer inversions, unlike the first-layer inversion where we use densely sampled frequency data as usual. The sparsely sampled frequencies together have the full wave number coverage for effective imaging. Combined use of dense and sparse sampling in frequency is a compromise between resolution and efficiency, as it reduces the number of iterations needed in layer-stripping inversion while still producing a good image. In the second scheme, we apply depth-dependent weights to model updates in order to improve the convergence in an iterative solution. The weighting is inversely proportional to the ray density variation along the depth and is mathematically equivalent to the application of an inverse Hessian matrix which sharpens the gradient vector for model updating. For real seismic data, we transfer point source shot records to line source records, by partial amplitude compensation and phase adjusting, before inputting it to the waveform tomography. We perform traveltime inversion to generate a reliable layered velocity model and then waveform tomography to produce a high-resolution image of the subsurface model through frequency domain iteration.

Citation: Wang, Y., and Y. Rao (2009), Reflection seismic waveform tomography, J. Geophys. Res., 114, B03304, doi:10.1029/ 2008JB005916.

\section{Introduction}

[2] This paper presents strategies and procedures for seismic waveform tomography with reflection geometry, where reflection seismic data are recorded at the earth's surface with limited source-receiver offsets. Waveform tomography has been used successfully on transmission data, such as crosshole seismic data [Pratt and Worthington, 1990; Song et al., 1995; Pratt and Shipp, 1999; Zhou and Greenhalgh, 2003; Pratt et al., 2004; Wang and Rao, 2006; Rao et al., 2006]. It also has been applied to wide-angle reflection/refraction seismic data with a certain degree of success [Bunks et al., 1995; Pratt et al., 1996; Ravaut et al., 2004; Operto et al., 2006; Bleibinhaus et al., 2007; Brenders and Pratt, 2007a, 2007b]. In addition, waveform tomography has been used for regional-scale studies on the crustal and upper mantle velocity structure, using scattering waves, surface waves and SH waves from either exploration seismics or broadband teleseismograms [Helmberger et al., 2001; Pollitz and Fletcher, 2005; Priestley et al., 2006]. However, there is little progress on its application to reflection seismic

\footnotetext{
${ }^{1}$ Centre for Reservoir Geophysics, Department of Earth Science and Engineering, Imperial College London, London, UK.
}

Copyright 2009 by the American Geophysical Union. 0148-0227/09/2008JB005916\$09.00 data with limited source-receiver offsets [Pratt et al., 1998; Hicks and Pratt, 2001; Shin and Min, 2006]. On the other hand, the seismic reflection method is a routine practice in the hydrocarbon exploration, as the data dominated by the precritical reflection energy, reflected back from subsurface contrasts in physical parameters, are well suited for seismic migration for the structural image. Therefore, it would be desirable if we are able to use these seismic reflection data to quantitatively extract the earth's physical parameters, which are essential for identifying different lithologies, different fracture characteristics, and even for indicating the hydrocarbon distribution directly.

[3] However, when applying waveform tomography to offset-limited reflection seismic data, it is difficult to recover long-wavelength variations in velocity, since reflection waveforms are highly sensitive to the contrasts in velocity (or impedance), and are not so sensitive to long-wavelength variations in velocity [Mora, 1988]. Snieder et al. [1989] explored the possibility of decoupling the smooth reference velocity and the short-wavelength variations by performing waveform fit in an alternating fashion. Hicks and Pratt [2001] suggested first to invert for the short-wavelength variation in velocity and then for long-wavelength variation with a suitable reparameterization to much fewer model parameters. In any case, it is common to use traveltime tomography to recover the long-wavelength velocity variation, 

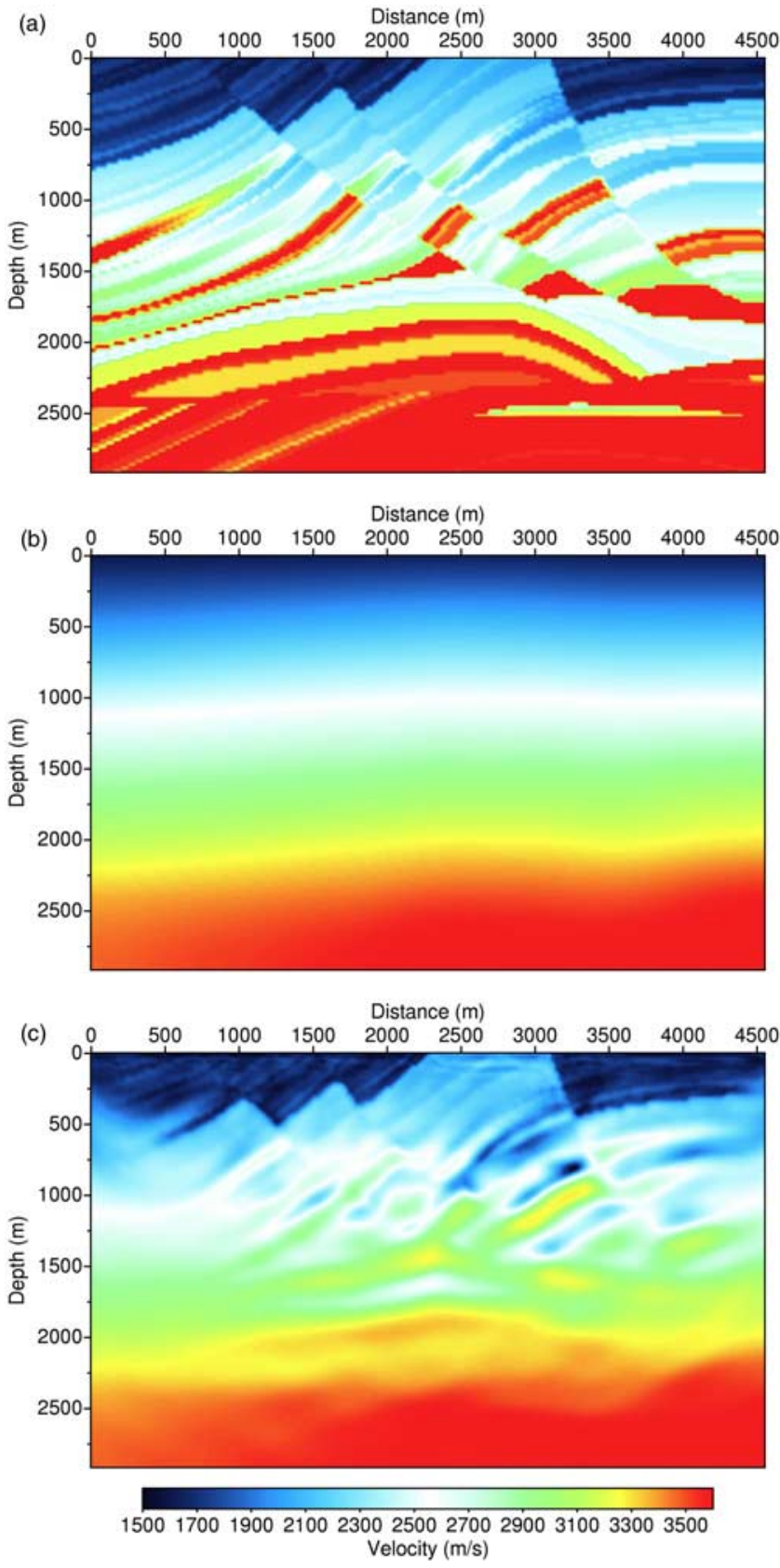

Figure 1. (a) The Marmousi velocity model. (b) The initial velocity model used in waveform tomography. (c) Waveform inversion image, in which only the top portion of the model is well reconstructed.

and serve as a good starting model for the iterative waveform inversion [Wang and Rao, 2006].

[4] Another difficulty, when using reflection seismic data in waveform tomography, is to recover the velocity variation in the deep part of the model. There are number of causes. First, because seismic reflection amplitudes decrease along with increased depth, near-surface waveforms dominate the data fitting in the inversion. In other words, near-surface velocity updates have much more influence on the data fitting than the deep-part updates. Second, ray density in the shallow part is much higher than that in the deep part of the model. Tomography is an integral along raypaths, on which data residuals are evenly distributed, therefore, high ray density leads to a large model update, and low ray density to a small velocity update in the iterative solution. To tackle the problem, we present two schemes in this paper. The first scheme is a layer-stripping procedure which makes the inversion focus on the reconstruction of the deep part of the model, by removing the top portion of the data from the inversion and keeping the top part of the model unchanged. The second scheme scales the velocity updates with weights according to the depth in the model.

[5] For real seismic data, we need to transform point source shot records to line source shot records, before input to waveform tomography. This is a partial compensation involving amplitude correction and phase adjusting, implemented in the time domain and frequency domain, respectively. For typical reflection seismic data in hydrocarbon exploration, we have no information less than $6 \mathrm{~Hz}$. Thus we perform reflection traveltime inversion to build a layered velocity model, and use it in turn as an initial model for waveform tomography. In waveform tomography, we use different frequency components of data from low to high frequencies in sequence, to gradually improve the resolution of the subsurface velocity model.

\section{Difficulty in Reflection Tomography}

[6] Seismic waveform tomography is implemented in the frequency domain [Pratt and Worthington, 1990; Pratt et al., 1998]. The objective function for the inverse problem is defined by data misfit as

$$
J(\mathbf{m})=\left[\mathbf{P}(\mathbf{m})-\mathbf{P}_{\mathrm{obs}}\right]^{H} \mathbf{C}_{\mathrm{D}}^{-1}\left[\mathbf{P}(\mathbf{m})-\mathbf{P}_{\mathrm{obs}}\right],
$$

where vector $\mathbf{m}$ is the velocity model to be inverted for, $\mathbf{P}(\mathbf{m})$ is a synthetic seismic response based on the estimated velocity model $\mathbf{m}, \mathbf{P}_{\text {obs }}$ is an observed seismic data set in the frequency domain, and $\mathbf{C}_{D}$ is the data covariance matrix with units of (data) $)^{2}$, describing the uncertainties in the observed data set. The superscript $H$ in equation (1) denotes the complex conjugate transpose. The frequency domain waveform tomography is performed iteratively. For each iteration, the inversion procedure may be divided into four steps [Song et al., 1995; Operto et al., 2006; Wang and Rao, 2006]: (1) for a given model estimate, calculating the synthetic wavefield $P_{0}\left(\mathbf{r} ; \mathbf{r}_{s}\right)$ at space position $\mathbf{r}$ corresponding to a source point at $\mathbf{r}_{s}$; (2) using the weighted data residual $\delta \hat{\mathbf{P}}=\mathbf{C}_{\mathrm{D}}^{-1} \delta \mathbf{P}$ as virtual sources to generate a so-called back-propagation wavefield $P_{\mathrm{b}}\left(\mathbf{r} ; \mathbf{r}_{s}\right) ;(3)$ cross correlation of the original wavefield $P_{0}\left(\mathbf{r} ; \mathbf{r}_{s}\right)$ and the backpropagation wavefield $P_{\mathrm{b}}\left(\mathbf{r} ; \mathbf{r}_{s}\right)$ to get the gradient direction $\gamma=\mathbf{C}_{\mathrm{M}} \hat{\gamma}$, where $\mathbf{C}_{\mathrm{M}}$ is the model covariance matrix with units of (model parameter) ${ }^{2}$; and (4) estimating the model update $\delta \mathbf{m}=-\alpha \gamma$, where $\alpha$ is the optimal step length that can be found by using the linear approximation or simply line search for a minimum of the objective function.

[7] For a detailed theoretical background about waveform tomography, readers may refer to Tarantola $[1984,1987$, 2005]. The frequency domain treatment can be found from Pratt and Worthington [1990] and Pratt et al. [1998].

[8] Figure 1a shows the Marmousi velocity model of size $4550 \times 2800 \mathrm{~m}$. We set up such a model with relatively narrow spatial extent for the purpose of testing the capacity 

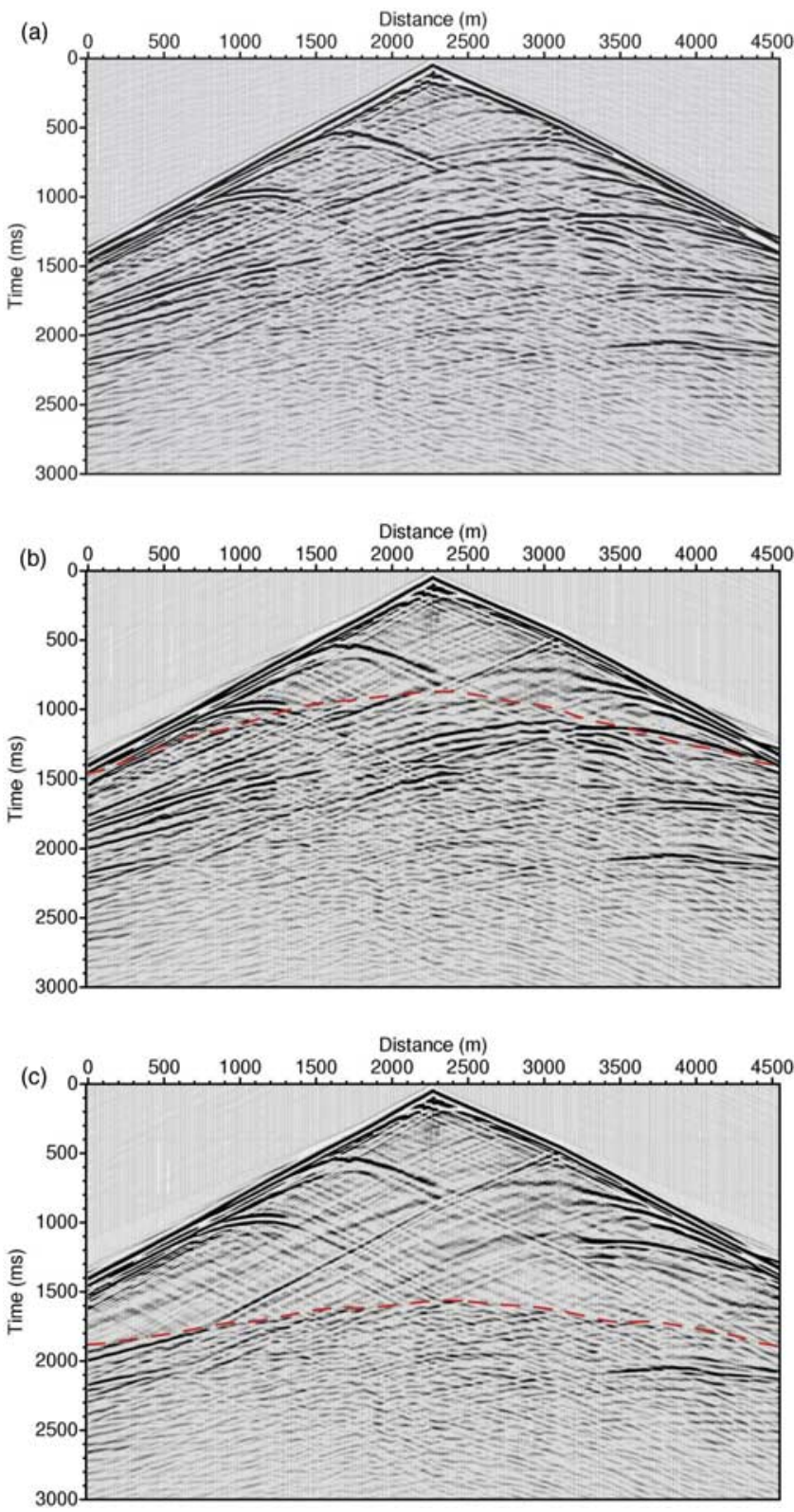

Figure 2. (a) A shot record generated from the true model. (b) A shot record in which the bottom portion (below the dashed curve) is the original data and the top portion (above the dashed curve) is generated from the first tomographic model. (c) A shot record combining the original data (below the dashed curve) and the synthetic (above the dashed curve) generated from the second tomographic model. The dashed curves in Figures $2 b$ and $2 c$ are the reflection traveltime curves from the auxiliary plane interfaces at depths 1000 and $2000 \mathrm{~m}$, respectively.

of seismic waveform tomography using reflection data with limited source-receiver offsets as in exploration seismology. In the frequency domain forward modeling and tomographic inversion, the velocity model is discretized into $12.5 \mathrm{~m}$ cells, and the wave equation is solved using a finite difference scheme [Alford et al., 1974; Kelly et al., 1976; Virieux, 1986; Levander, 1988; Pratt, 1990; Song et al., 1995; Stekl and Pratt, 1998; Pratt, 1999; Min et al., 2000].
[9] Figure $1 \mathrm{~b}$ shows the initial model used in the iterative waveform tomography. It is a smooth version of the true velocity model and is obtained by low-pass filtering with cutoff wave number of $5 \mathrm{~km}^{-1}$. As a reference, the discretized velocity model with cell size of $12.5 \mathrm{~m}$ has the Nyquist wave number $k_{N y q}=40 \mathrm{~km}^{-1}$. Thus the initial model presents the long-wavelength background variation in the velocity model, removing one of the possible causes of problems in inversion convergence.

[10] Figure 1c shows the velocity image obtained from waveform tomography. The frequencies used in inversion are within the range from 2.7 to $30.0 \mathrm{~Hz}$ with $0.3 \mathrm{~Hz}$ sample interval. The maximum frequency $f_{\max }=30 \mathrm{~Hz}$ is due to the limitation of our frequency domain finite difference modeling:

$$
f_{\max }=\frac{c_{\text {min }}}{4 \Delta x},
$$

where the minimum velocity $c_{\min }=1500 \mathrm{~m} / \mathrm{s}$, and cell size $\Delta x=12.5 \mathrm{~m}$. Because the data used are seismic reflection data recorded with limited source-receiver offsets, we can only obtain a well-constructed image of the topmost portion of the model. In the following two sections, we will present two schemes to overcome this problem in reflection seismic waveform tomography.

\section{Layer-Stripping Scheme for Reflection Tomography}

\subsection{Layer-Stripping Implementation}

[11] In a shot gather recorded at the earth surface, shallow reflections are stronger than deep reflections and have more contribution to the data misfit in the inversion's objective function. Deep reflections with weak energy cannot be properly used in a waveform inversion to reconstruct a subsurface image. Thus, a layer-stripping scheme seems appropriate to deal with shallow and deep reflections in sequence. We start the waveform inversion for the whole model, as shown in Figure 1c. After that, we assume that the topmost part of the model is well imaged and concentrate on the reconstruction of the rest of the model. Then, we assume the second layer of the model is also well imaged and focus on the improvement of further deeper portions.

[12] Therefore, in each inversion of the layer-stripping implementation, we divide the velocity model into basically two parts: The top part is well reconstructed, and the bottom part needs further update by waveform tomography. Accordingly, the input to waveform tomography is a combination of the original shot record and a synthetic record: the top part is the synthetic generated from the well-reconstructed top portion of the model, and the rest is the original seismic data. In waveform tomography, where we update the model parameters on the basis of the data residual back propagation, we also keep the top portion of the model unchanged, and restrict our model update to the bottom part of the model.

[13] The Marmousi model (Figure 1) is used again to demonstrate the layer-stripping scheme for the reflection waveform tomography. There are 362 source/receiver points spreading along the surface from 0 to $4550 \mathrm{~m}$, with interval $12.5 \mathrm{~m}$. A seismic trace is sampled to 2001 points with time interval of $2 \mathrm{~ms}$, and the total length of a trace is $4 \mathrm{~s}$. Figure 2a 

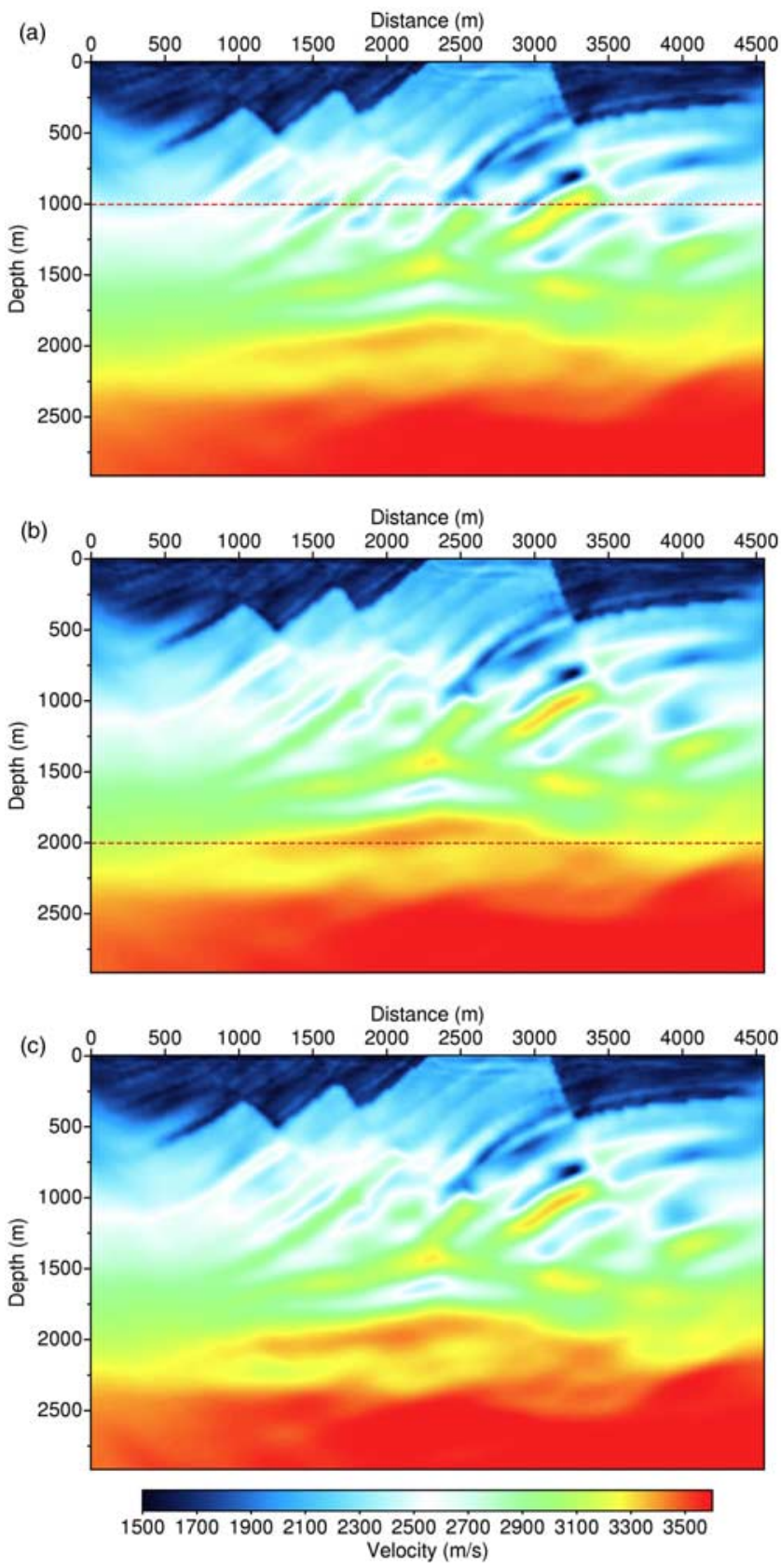

Figure 3. (a) The first tomographic image, in which only the top portion of the model is well imaged. (b) Tomographic inversion focusing on the model part below depth $1000 \mathrm{~m}$, in which the model above depth $2000 \mathrm{~m}$ is well imaged. (c) Tomographic inversion focusing on the model part below depth $2000 \mathrm{~m}$.

is an example shot record generated from the true velocity model of Figure 1a, and is one of the shot records input to the first-time waveform inversion.

[14] In layer stripping to improve the model image in the deep part, we set up an auxiliary plane boundary at depth $1000 \mathrm{~m}$. Figure $2 \mathrm{~b}$ is a shot record in which the top portion of the original data is replaced with the synthetic generated from the first waveform tomography model (Figure 1c). The dashed curve is the reflection traveltime curve from the auxiliary boundary at depth $1000 \mathrm{~m}$, calculated using a bending ray-tracing method [Wang and Houseman, 1995; Wang, 2003]. We use the combined shot gathers such as Figure $2 \mathrm{~b}$ for the second waveform inversion. Similarly, Figure $2 \mathrm{c}$ is a combined shot gather showing a dashed curve of reflection traveltimes from the auxiliary plane boundary at depth $2000 \mathrm{~m}$ in the model. We use this combined shot record as the input to the third waveform inversion.

[15] Figure 3 demonstrates successive improvements in the model image. After the first waveform tomography (Figure 3a), we assume that the top portion of the model is well imaged and focus on improving the part below the auxiliary plane boundary at $1000 \mathrm{~m}$. After the second inversion (Figure 3b), the model above depth $2000 \mathrm{~m}$ is well imaged. Then we focus on improving the bottom part of the model below the depth $2000 \mathrm{~m}$. Figure 3c shows the final image from our layer-stripping waveform tomography.

[16] In each inversion, we keep the velocities unchanged above an auxiliary boundary, and implement the model update only to the part below either 1000 or $2000 \mathrm{~m}$ depth. This is equivalent to giving a zero weight to the velocity update above the boundary and a nonzero weight to the velocity update below. As the data residual in an inversion is spread to the model updates averagely along a raypath, when a zero weight is given to some updates, a nonzero weight for the rest should be larger than the unity. For the second-time inversion to update a $2 / 3$ portion of the model, the nonzero weight is 1.5 . For the third-time inversion to update a $1 / 3$ portion of the model, the nonzero weight is 3.0.

[17] Figure 4a displays a synthetic shot record, generated from the first inversion model (Figure 3a). Figure $4 \mathrm{~b}$ plots the difference between the synthetic records generated from the first inversion model (Figure 3a) and the second inversion model (Figure 3b). Figure $4 \mathrm{c}$ is the difference between the synthetic records generated from the second inversion model (Figure $3 \mathrm{~b}$ ) and the third inversion model (Figure 3c). Note that different amplitude scales are used in the latter two plots, to reveal the very weak change in waveform amplitudes.

\subsection{Frequency Selection Strategy}

[18] As the number of inversions in a layer-stripping scheme is proportional to the number of layers to be stripped, the computation time is linearly proportional to the number of layers and also the total number of temporal frequencies used in the inversion. We try now to use a frequency selection strategy to reduce the number of frequencies needed in waveform tomography. In reflection geometry, source-receiver pairs vary with different offsets and move along the surface. Sirgue and Pratt [2004] suggested make an optimal use of different offsets in the reflection experiment to create a variety of plane wave imaging directions, and proposed a strategy for the frequency discretization as

$$
f_{n+1}=\frac{f_{n}}{\alpha},
$$

where $f_{n}$ is the frequency previously used, $f_{n}+1$ is a new frequency for inversion, and $\alpha=z / \sqrt{h_{\max }^{2}+z^{2}}$ is the cosine of the reflection angle, corresponding to the maximum value 

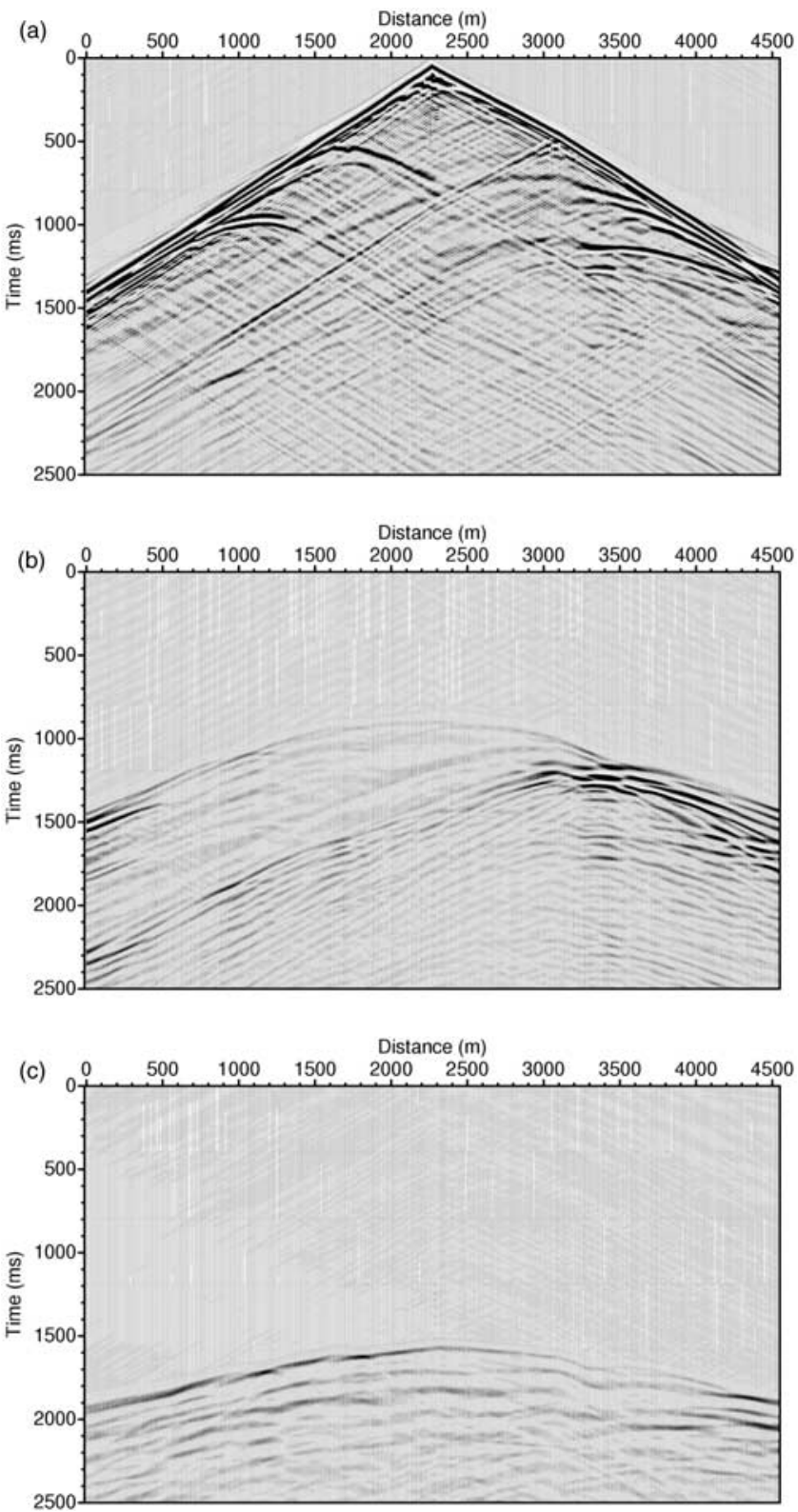

Figure 4. (a) A synthetic shot record generated from the first inversion model (Figure 3a). (b) Difference between the shot records generated from the second and first inversion models (Figures 3b and 3a). (c) Difference between the shot records generated from the third and second inversion models (Figures $3 c$ and $3 b$ ). Note that different scales are used to plot the wave amplitudes in these three plots to reveal weak amplitude changes in deeper-layer inversions.

of the half source-receiver offset $h_{\max }$ and the target depth $z$. This strategy has been used recently by Brenders and Pratt [2007b].

[19] For the Marmousi model here with $h_{\max }=2275 \mathrm{~m}$, $z=2800 \mathrm{~m}$ and $\alpha \approx 0.776$, we may select the following 11 frequencies: $2.7,3.4,4.4,5.7,7.3,9.4,12.0,15.5,20.0$, $25.7,30.0 \mathrm{~Hz}$. In this list, we include the dominant frequency $20 \mathrm{~Hz}$ in the inversion, and set the last frequency as $f_{\max }=30 \mathrm{~Hz}$, because of the limitation of finite difference modeling as shown in equation (2), which is less than the predicted value of $33.1 \mathrm{~Hz}$. This strategy eliminates some frequencies but has a continuous coverage on vertical wave number $k_{z}$, as

$$
k_{z_{\min }}\left(f_{n+1}\right)=k_{z_{\max }}\left(f_{n}\right),
$$

where

$$
k_{z_{\max }}=\frac{f}{\frac{1}{2} c_{0}}, k_{z_{\min }}=\frac{\alpha f}{\frac{1}{2} c_{0}}
$$

and $c_{0} / 2$ is half of the background velocity [Wu and Toksöz, 1987]. Figure 5 illustrates the wave number coverage corresponding to the selected frequencies in waveform tomography.

[20] On the other hand, if we assume there is no sourcereceiver offset coverage to be exploited, we need to have adequate wave number samples in order to obtain a sufficiently good image in the model space. In this case the sampling rate should satisfy the antialiasing condition, $\Delta k_{z} \leq$ $1 / z_{\max }$, where $z_{\max }$ is the maximum depth to be imaged, and $\Delta k_{z}$ is the sampling rate of the vertical wave number. Given the minimum value of the vertical wave number at frequency $f$ by equation (5), we have the difference between two neighboring wave number samples as

$$
\Delta k_{z} \equiv k_{z_{\min }}(f+\Delta f)-k_{z_{\min }}(f)=\frac{2 \alpha \Delta f}{c_{0}} \leq \frac{1}{z_{\max }} .
$$

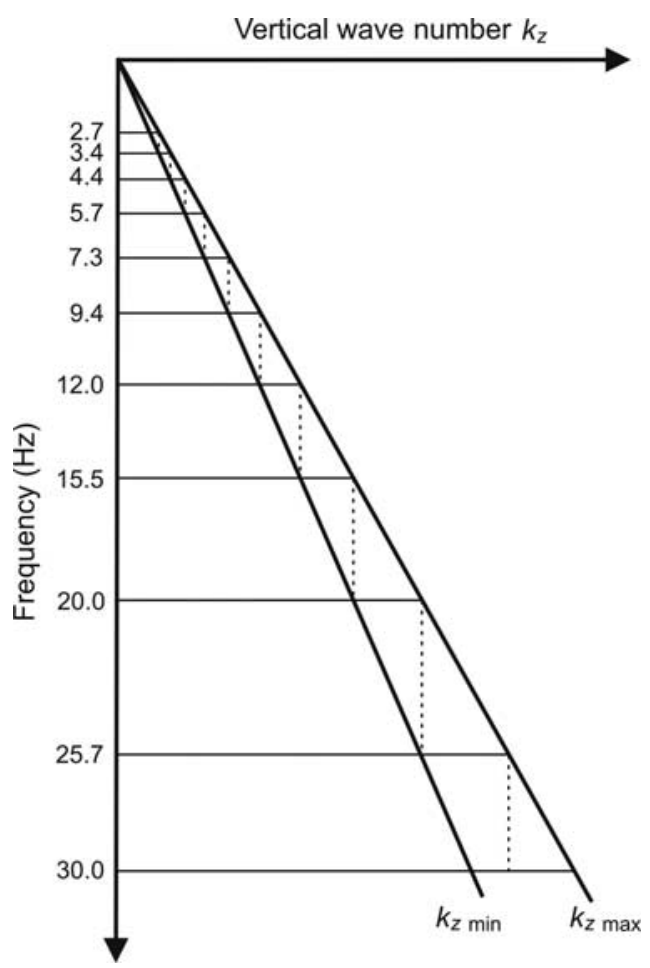

Figure 5. The coverage of the vertical wave numbers corresponding to 11 selected frequencies for waveform tomography. 


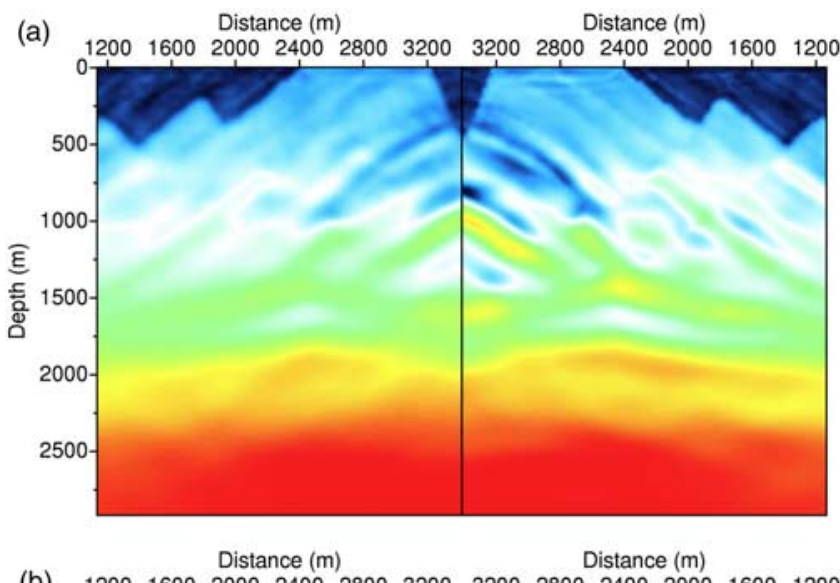

(b) $1200 \quad 1600 \quad 2000 \quad 2400 \quad 2800 \quad 3200 \quad 3200 \quad 2800 \quad 2400 \quad 2000 \quad 1600 \quad 1200$

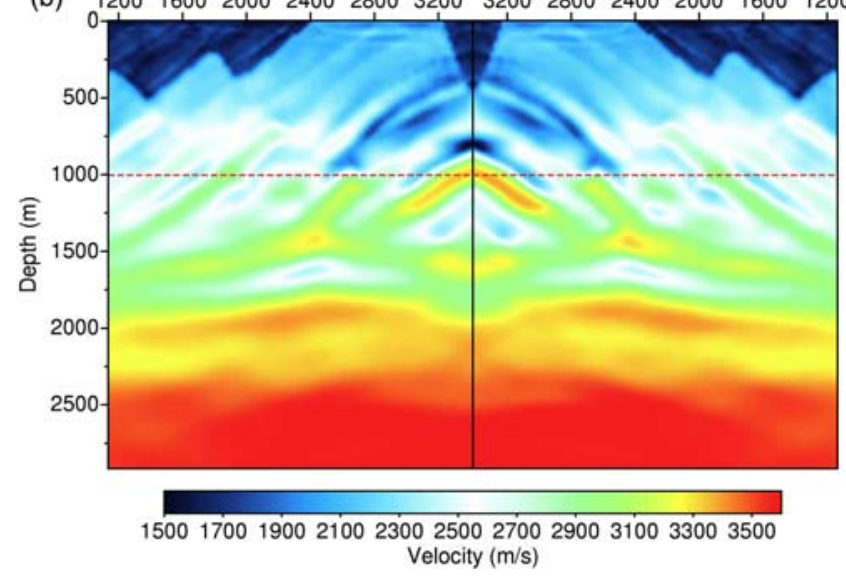

Figure 6. (a) Comparison of two frequency selection schemes: on the left is the inversion result of 11 sparsely selected frequencies, and on the right is the mirror image of the waveform tomography result with the full frequency range between 2.7 and $30 \mathrm{~Hz}$ with a sampling interval of $0.3 \mathrm{~Hz}$. (b) Comparison of two frequency selection schemes in a layer-stripping procedure. On the left, the first inversion uses the full frequency range, but the rest uses 11 sparsely sampled frequencies. On the right, each time inversion uses the full frequency range.

We finally obtain the following antialiasing condition for frequency sampling:

$$
\Delta f \leq \frac{c_{0}}{2 \alpha z_{\max }} .
$$

In this example, we set up $\Delta f=0.3 \mathrm{~Hz}$.

[21] Figure 6a compares these two frequency selection schemes, where the left side is the inversion result obtained from the sparsely selected 11 frequencies, and the right side is a (mirror) image of waveform tomography with the full frequency range with a sampling interval of $0.3 \mathrm{~Hz}$. For each frequency, we perform 10 iterations of the inversion. The comparison shows that waveform tomography using 11 selected frequencies has produced a faint image. This comparison actually suggests that the total number of iterations plays an important role in the inversion. Although 11 selected frequencies have full wave number coverage, the total number of iterations in the inversion is $110(=10 \times 11)$. With densely sampled frequencies, the wave number coverage was not increased at all, but the total number of iterations $(10 \times 92=920)$ has been increased significantly in the inversion. For this synthetic example, computation time needed for the inversion of a single iteration and a single frequency is about $5.2 \mathrm{~min}$, running with a single CPU. Therefore, 920 iterations needs about $80 \mathrm{~h}$, and 110 iterations needs about $9 \mathrm{~h}$. Hence, time saving is significant with this frequency selection scheme.

[22] Assuming we have used densely sampled frequencies in the first inversion and produced a better constructed image at the topmost portion of the model and a reasonable image for the deeper part of the model, Figure $6 \mathrm{~b}$ compares two frequency sampling strategies for the second inversion (below the red dashed line). The left-hand side is the inversion result using 11 selected frequencies, and the righthand side is the (mirror) image generated by using densely sampled frequencies. We can see that the two results are quite similar from the auxiliary plane interface of $1000 \mathrm{~m}$ downward.

[23] Therefore, to afford efficiency in a layer-stripping procedure, we make a compromise by using densely sampled frequencies with a constant sampling rate for the first time of waveform tomography, and then using selective frequencies with much sparse sampling for subsequent inversions to improve the image of deeper portion of the model. The computational time is no longer strictly proportional to the number of layers in stripping. For all subsequent inversions other than the first inversion, the computational time is just fractional. Thus, the efficiency of such a compromised layer-stripping scheme is affordable.

\section{Weighted Updating Scheme for Reflection Tomography}

[24] In this section, instead of using a layer-stripping approach, we present another scheme that can invert for the entire velocity model at once, in which we apply depthdependent weighting factors to velocity updating in the inversion.

[25] By definition, tomography is an integral along the raypath; that is, data residuals are spread evenly along the raypath. The residual projected to a cell is then linearly related to the model update of the cell, and thus the model update depends upon the total length of ray segments within the cell, i.e., the ray density. Since the ray density in the shallow depth is much higher than that in deeper parts of the model, the model update in the shallow depth is much higher than that in the deep portion of the model. This is a underlying rationale for using depth-dependent weights in model updating.

[26] In the gradient inversion method, the objective function is minimized by updating the model vector along the opposite direction to the gradient:

$$
\mathbf{m}^{(\ell+1)}=\mathbf{m}^{(\ell)}-\alpha \boldsymbol{\gamma}
$$

Pratt et al. [1998] pointed out that the application of an inverse Hessian matrix would sharpen or focus the gradient 

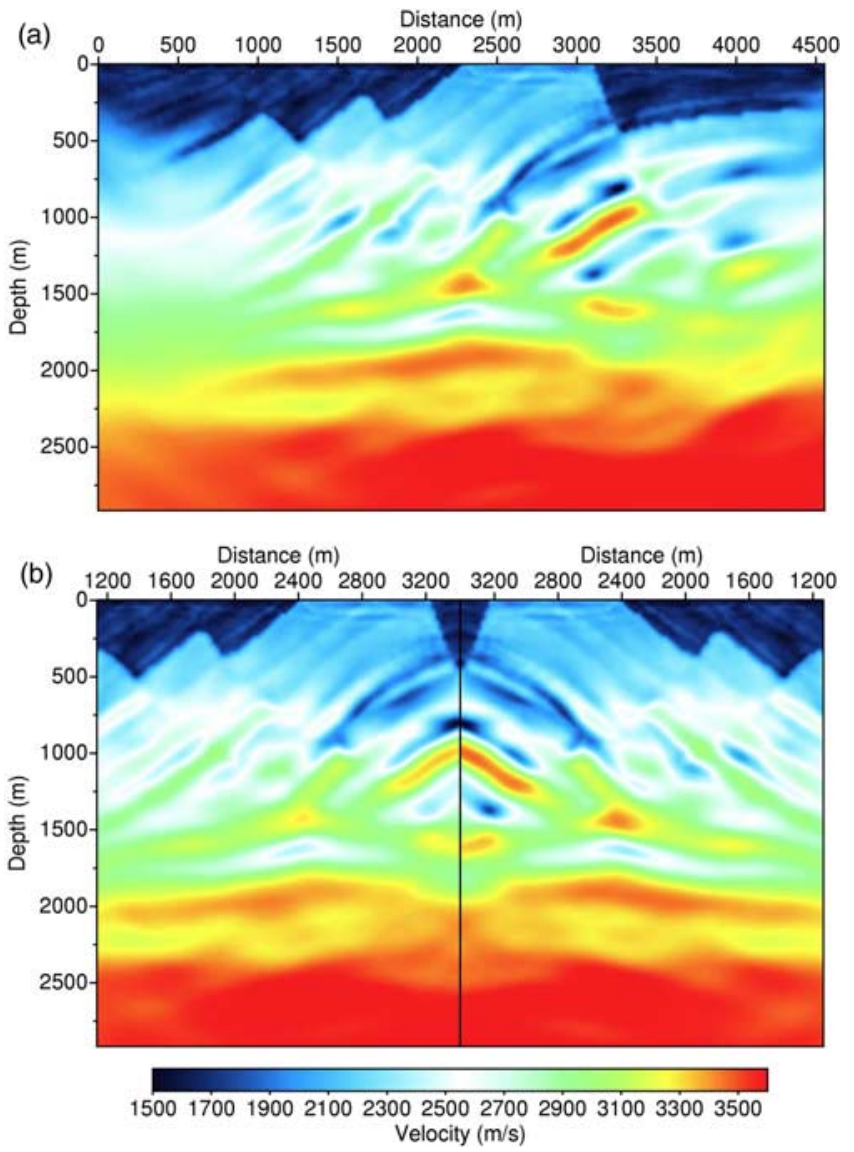

Figure 7. (a) Tomographic image of waveform inversion with scaled model updates. (b) Comparison of two inversion schemes: the left side is the inversion result using the layerstripping scheme, and the right side is the (mirror) image of waveform tomography with scaled model updates.

vector $\gamma$ and could improve the convergence rate in an iterative solution:

$$
\mathbf{m}^{(\ell+1)}=\mathbf{m}^{(\ell)}-\alpha \mathbf{H}^{-1} \gamma
$$

where $\mathbf{H}$ is the Hessian matrix,

$$
\mathbf{H} \approx \operatorname{Re}\left\{\mathbf{J}^{T} \mathbf{J}^{*}\right\}
$$

given in terms of matrix $\mathbf{J}$, the Fréchet derivatives of seismic wavefield with respect to the model elements: $J_{i j}=$ $\partial P_{i} / \partial m_{j}$, for $i=0,1, \ldots, N-1, j=0,1, \ldots, M-1$. The Hessian matrix $\mathbf{H}$ in (10) is an approximation. An accurate Hessian matrix of second derivative of the objective function with respect to the model parameters involves not only the multiplication of Fréchet matrix and its transpose but also the derivative of the Fréchet matrix. The latter is generally expensive to compute but is small if the residual is small or the forward equation is quasi-linear, and is often dropped off in practical application [Tarantola, 1987]. Pratt et al. [1998] showed that the diagonal of the approximate Hessian matrix $\mathbf{H}$ is dominant compared to other components, and that its inverse matrix could be used to scale the images. Shin et al. [2001] also addressed the issue whereby the Hessian matrix in the Gauss-Newton method plays a scaling role in the steepest-descent direction.

[27] By definition, the wavefield is given by

$$
P(\mathbf{r}, \omega)=S\left(\mathbf{r}_{s}, \omega\right) G\left(\mathbf{r}, \mathbf{r}_{s}, \omega\right)
$$

where $\mathbf{r}$ is the position vector, $\mathbf{r}_{S}$ is the source position, $S\left(\mathbf{r}_{s}, \omega\right)$ is the source amplitude at the frequency $\omega$, and $G\left(\mathbf{r}, \mathbf{r}_{s}, \omega\right)$ is Green's function in the frequency domain. In a homogeneous medium, the frequency domain, free space, two-dimensional (2-D) Green's function is

$$
G_{2 D}\left(\mathbf{r}, \mathbf{r}_{s}, \omega\right)=\left(\frac{-\mathrm{i} c}{8 \pi \omega R}\right)^{1 / 2} \exp \left[-\mathrm{i} \omega \frac{R}{c}\right]
$$

where $R=\left|\mathbf{r}-\mathbf{r}_{s}\right|$ is the distance from the source, and $c$ is the acoustic velocity of the medium. Considering equations (10)-(12), we can find that the main diagonal elements of the inverse Hessian matrix $\mathbf{H}^{-1}$ have the following behavior:

$$
\left[\mathbf{H}^{-1}\right]_{j j} \propto R c
$$

since $J_{i j} \propto(R c)^{-1 / 2}$. The analysis above leads us to a scaling scheme for the model updates in reflection waveform inversion as the following:

$$
\mathbf{m}^{(\ell+1)}=\mathbf{m}^{(\ell)}-\alpha \beta(z) \gamma,
$$

where $\beta(z)$ is a depth $(z)$ dependent scaling factor, $\beta(z) \propto$ $z c(z)$. As pointed out by Pratt et al. [1998], this scaling factor effectively compensates the geometrical spreading effect of the waveform amplitudes in the inversion.

[28] In the inversion example shown in Figure 7a, we use the scaling factor as

$$
\beta(z)=1.0+0.2 \times 10^{-6} z c(z) .
$$

For depth $z=0,1000,2000,2500$ and $2750 \mathrm{~m}$ with corresponding average velocity $c(\mathrm{z})=1500,2000,2500$, 3000 and $3600 \mathrm{~m} / \mathrm{s}$, we use the scale factor $\beta(z)=1.0,1.4$, 2.0, 2.5 and 3.0, respectively.

[29] Figure $7 \mathrm{~b}$ compares tomographic images of the layer-stripping scheme and depth-dependent scaling schemes directly. The latter scheme produces a marginally better image. For example, the low- and high-velocity layers in depth between 750 and $2000 \mathrm{~m}$ are much sharper than that in the image obtained from the layer-stripping scheme. Although both schemes are capable of producing a satisfactory result, the weighting scheme can invert the entire velocity model at once, and thus greatly reduces the computation time, in contrast to the layer-stripping scheme.

[30] We have also conducted inversion tests on noisy data. Using the synthetic data with random noise, both the layerstripping and the weighting schemes produce results that are very close to the inversion results for noise-free synthetic data. This is because the noise is randomly distributed in the time domain. In the frequency domain, the noise becomes ambient with a white spectrum. In other words, the frequency 


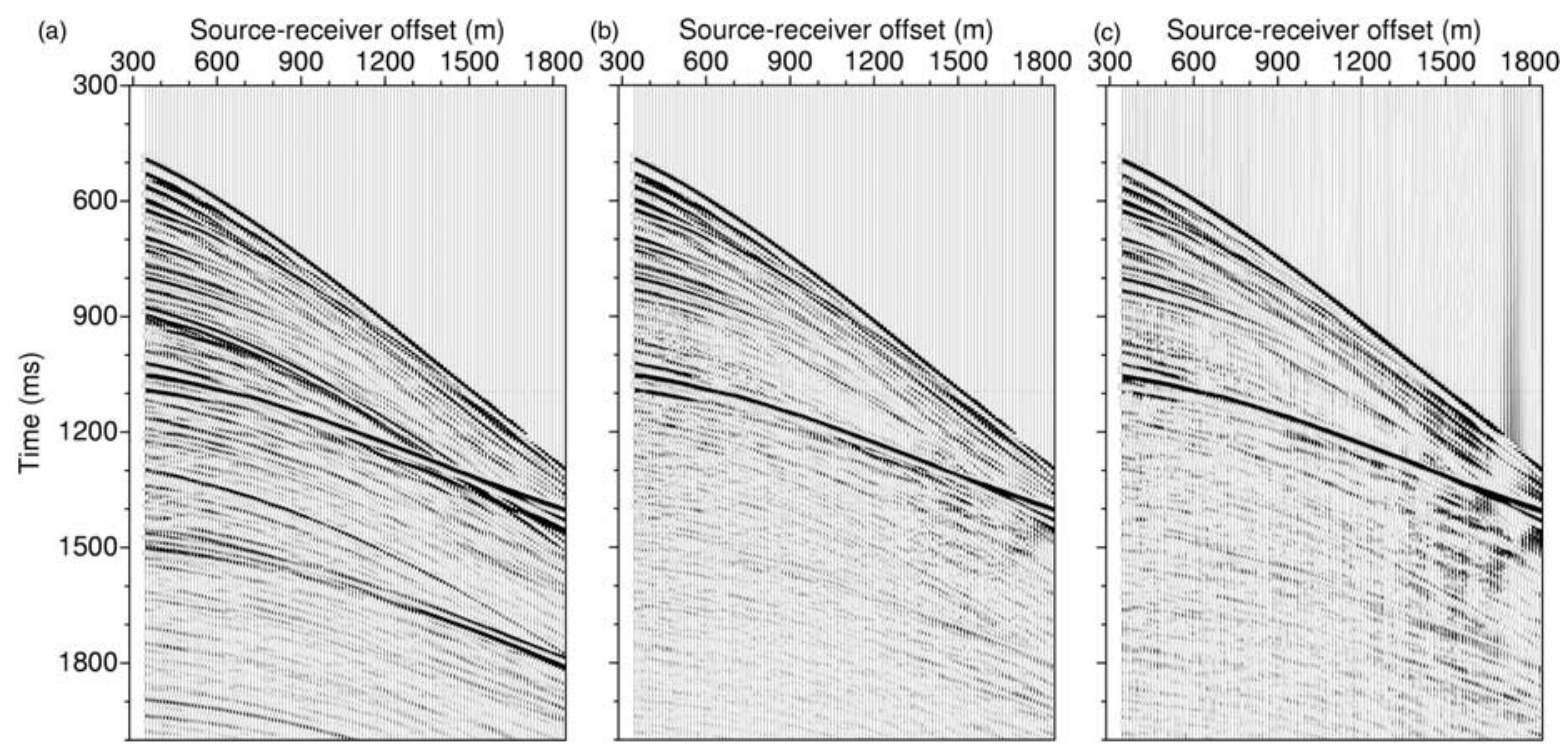

Figure 8. A marine seismic data example. (a) A sample shot record with 120 traces. (b) The shot record after multiple attenuation. (c) The same shot record after partial compensation.

domain inversion is not affected by random noise that is spectrally white.

[31] In practice however, real seismic data contain noise that is seldom distributed randomly and hardly has a white spectrum. A group of frequencies is then used as follows simultaneously in each iterative inversion, to suppress the noise effect in the frequency domain inversion, following Pratt and Shipp [1999], Wang and Rao [2006], and Brenders and Pratt [2007a].

\section{Strategies for Real Data Application}

[32] We now discuss the strategies for application of waveform tomography to a real, marine seismic data set. Figure $8 \mathrm{a}$ is a sample shot record, consisting of 120 traces with a minimum source-receiver offset of $337.5 \mathrm{~m}$ and a maximum source-receiver offset of $1825 \mathrm{~m}$. We investigate the feasibility of reflection seismic tomography within such a narrow source-receiver offset range.

\subsection{Free Surface Multiple Attenuation}

[33] The current waveform tomography code does not include free surface multiples, as we use an absorbing boundary condition at the free surface in the forward calculation. Including seismic multiples bouncing back and forward within the water layer in the tomographic inversion will increase the nonlinearity of the problem. As the number of multiples increases, the errors in model (and in turn in synthetics) will also increase. We use a narrow-offset shot record in multiple attenuation, also to avoid the wide-angle refraction of the water bottom and their multiples, as the current methodology for free surface multiple prediction cannot properly model the refraction multiples. As marked in Figure 8a, the most difficult part of multiple attenuation is where the refraction wave just starts appearing. Figure $8 \mathrm{~b}$ displays the shot record after free surface multiple attenuation, using a multiple prediction through the inversion (MPI) method presented by Wang [2004, 2007]. The real shot record is generated by a point source, but Figure $8 \mathrm{c}$ is an equivalent line source shot gather, after partial compensation as follows.

\subsection{Partial Compensation}

[34] Before input to waveform tomography, a shot record of real seismic data needs to be partially compensated, to become a gather generated from a line source. Equation (12) is the 2-D Green's function. For a three-dimensional (3-D) case, Green's function is

$$
G_{3 D}\left(\mathbf{r}, \mathbf{r}_{s}, \omega\right)=\frac{1}{4 \pi R} \exp \left[-\mathrm{i} \omega \frac{R}{c}\right]
$$

Comparing the 2-D and 3-D Green's functions, we obtain the partial compensation operator as

$$
W=\sqrt{\frac{2 \pi R c}{\mathrm{i} \omega}} .
$$

In the time domain, the operator $W$ shows the following behavior (in the far field):

$$
W(t)=D_{-1 / 2}(t) \sqrt{2 \pi R c},
$$

where $D_{-1 / 2}(t)$ is a half integrator, defined as the inverse Fourier transform of $(\mathrm{i} \omega)^{-1 / 2}$ [Deregowski and Brown, 1983]. For a narrow-offset, reflection geometry, we assume $2 R \propto c t$ and obtain

$$
W(t) \propto D_{-1 / 2}(t) c(t) \sqrt{t}
$$

We implement this partial compensation in two steps: applying a scale factor $c(t) \sqrt{t}$ in the time domain, and multiplying the operator $(\mathrm{i} \omega)^{-1 / 2}$ in the frequency domain.

[35] Figure 9a is a brute stack of the raw marine seismic data, Figure $9 \mathrm{~b}$ is the stack section of the seismic data set after multiple attenuation, whereas Figure 9c is the same 

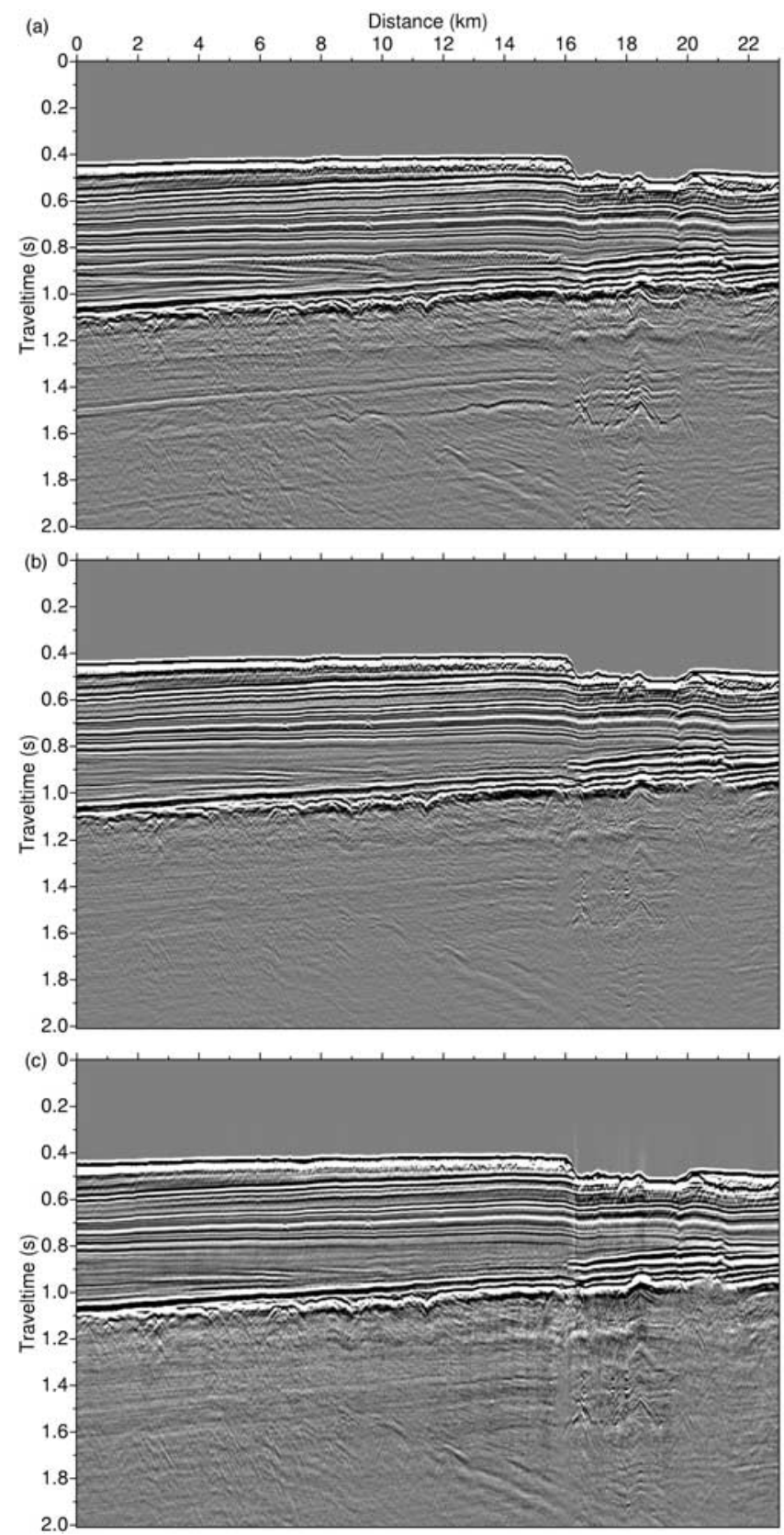

Figure 9. A marine seismic data example. (a) The stack section of the marine seismic data set without multiple attenuation. (b) The stack section of the marine seismic data set after multiple attenuation. (c) The same stack section with amplitude and phase compensations applied to the shot records before stacking. The compensations to the shot records make the original point sources become the equivalent line sources before they are used in waveform tomography.

stack section after applying the partial compensation to the shot records. Figure 10 closely compares a seismic trace from a point source (solid curve) and a trace after partial compensation (dash curve). With the application of the operator $(i \omega)^{-1 / 2}$, wavelets in the trace from an equivalent line source are broader than those actually generated from a point source.
[36] Alternatively, one could use a 2.5-D wave modeling and inversion scheme for waveform tomography. But as it involves integration along the infinite line source direction that is perpendicular to the source-receiver profile, a 2.5-D scheme would take a much longer running time, compared to the 2-D wave modeling and inversion [Song and Williamson, 1995].

[37] The frequency $\omega$ can also be complex valued, to include an exponential function either for the amplitude attenuation or compensation. The attenuation used in wavefield $P(t)$ is for suppressing the wrap-around effect in the Fourier transform domain. An opposite sign with an exponential increase in $P(t)$ could also be used to boost the energy of deep reflections. In the latter case, the real data should be balanced with an automatic gain control.

\subsection{Waveform Tomography}

[38] In the reflection seismic data, there is no significant energy recorded at low frequencies less than $6.5 \mathrm{~Hz}$. We perform a traveltime inversion to generate the initial velocity model for the iterative waveform tomography. Referring to the stack section (Figure 9), we pick the traveltimes of two reflections from the prestack seismic data, and run a traveltime inversion to generate a layered velocity model with two interfaces (Figure 11a). The first layer is a water layer with velocity $1500 \mathrm{~m} / \mathrm{s}$. The second layer has a linear velocity of $2200 \mathrm{~m} / \mathrm{s}$ at one end and $2000 \mathrm{~m} / \mathrm{s}$ at distance $23 \mathrm{~km}$.

[39] In the frequency domain waveform tomography, we use a group of three frequencies simultaneously in each

(a)

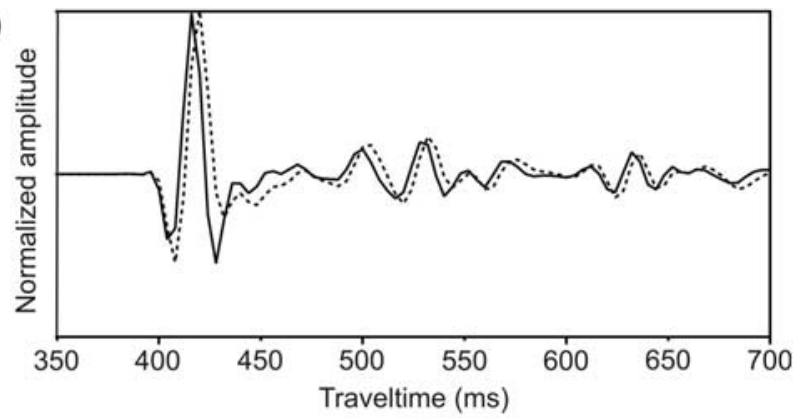

(b)

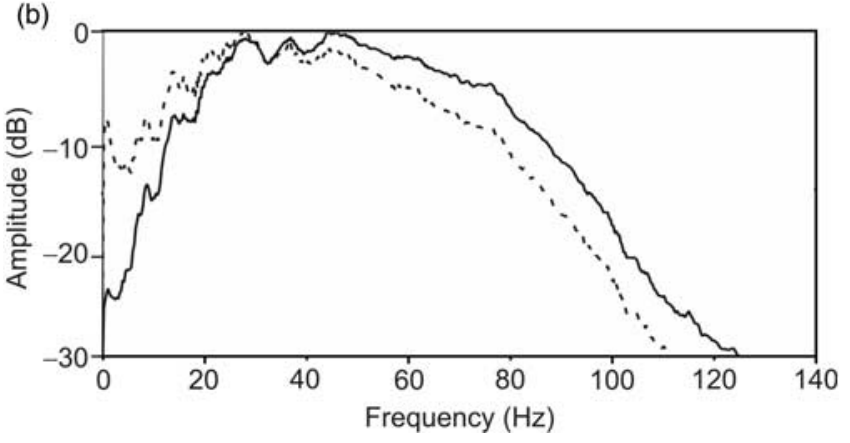

Figure 10. (a) Comparison between a seismic trace from a point source (solid curve) and the trace after partial compensation (dashed curve). Wavelets in a trace from a line source (i.e., after partial compensation) are broader than those from a point source. (b) Comparison of amplitude spectra of seismic traces from a point source and a line source. 

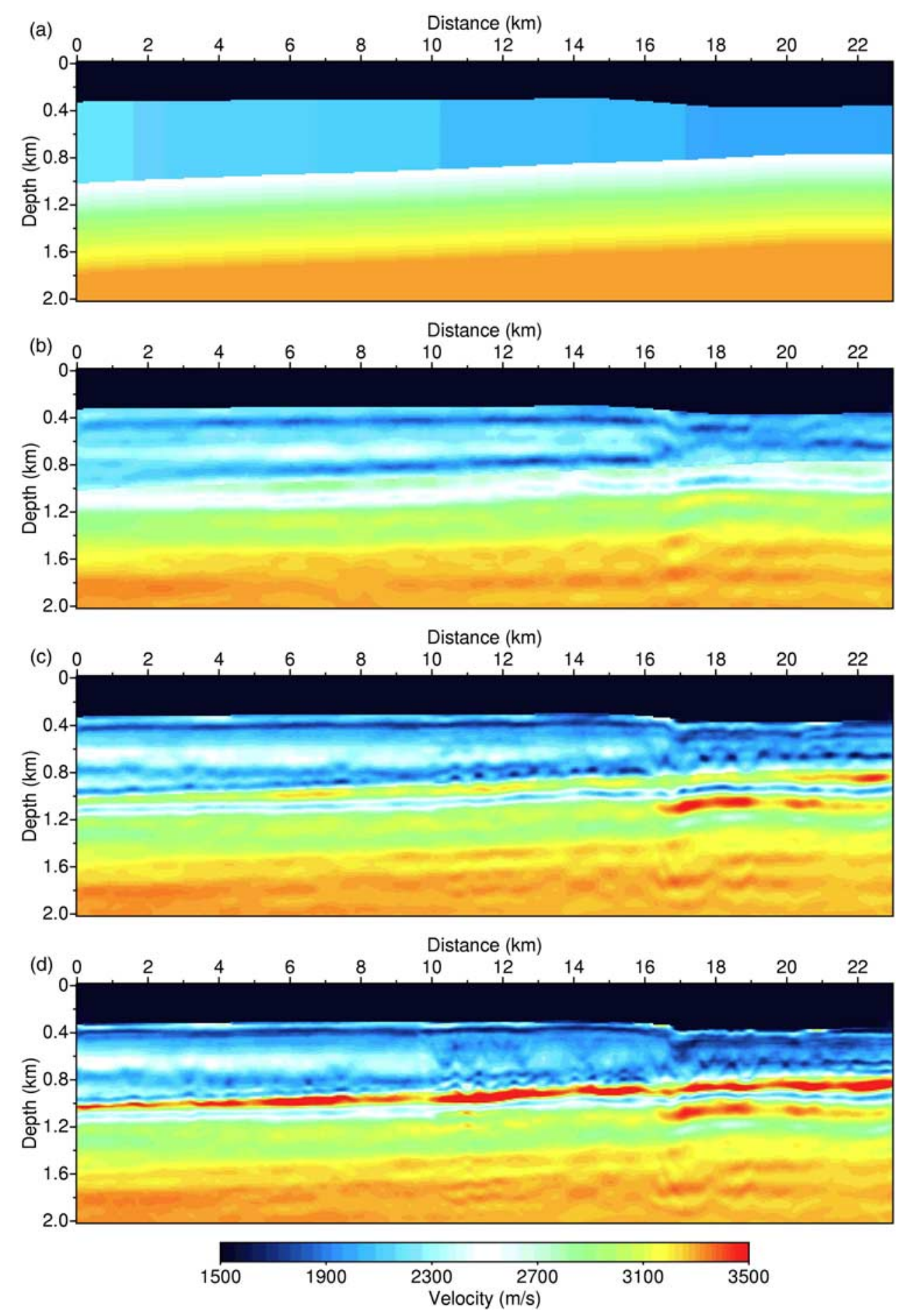

Figure 11. Waveform tomography. (a) The initial velocity model built from traveltime tomography. (b) The velocity model of waveform tomography using frequencies in the range of $6.9-7.5 \mathrm{~Hz}$. (c) The velocity model of waveform tomography using frequencies in the range of $6.9-13.8 \mathrm{~Hz}$. (d) The final velocity model obtained from the waveform tomography using all frequencies in the range of $6.9-30 \mathrm{~Hz}$.

iterative inversion [Pratt and Shipp, 1999; Wang and Rao, 2006; Brenders and Pratt, 2007a]. Using a group of neighboring frequencies in the input can suppress the noise effect in the real data, and also more data samples used in an inversion means a much better determined inverse problem. In this example, there are 26 groups of frequencies in total in the range of $6.9-30 \mathrm{~Hz}$ with an interval of $0.3 \mathrm{~Hz}$. The first group includes frequencies $6.9,7.2$ and $7.5 \mathrm{~Hz}$, and the last 29.4, 29.7 and $30 \mathrm{~Hz}$. Figures $11 \mathrm{~b}$ and $11 \mathrm{c}$ are the velocity models from waveform tomography using frequencies in the ranges of $6.9-7.5 \mathrm{~Hz}$ and $6.9-13.8 \mathrm{~Hz}$, respectively, where Figure $11 \mathrm{~d}$ is the final velocity model obtained from waveform tomography using all frequencies in the range. 

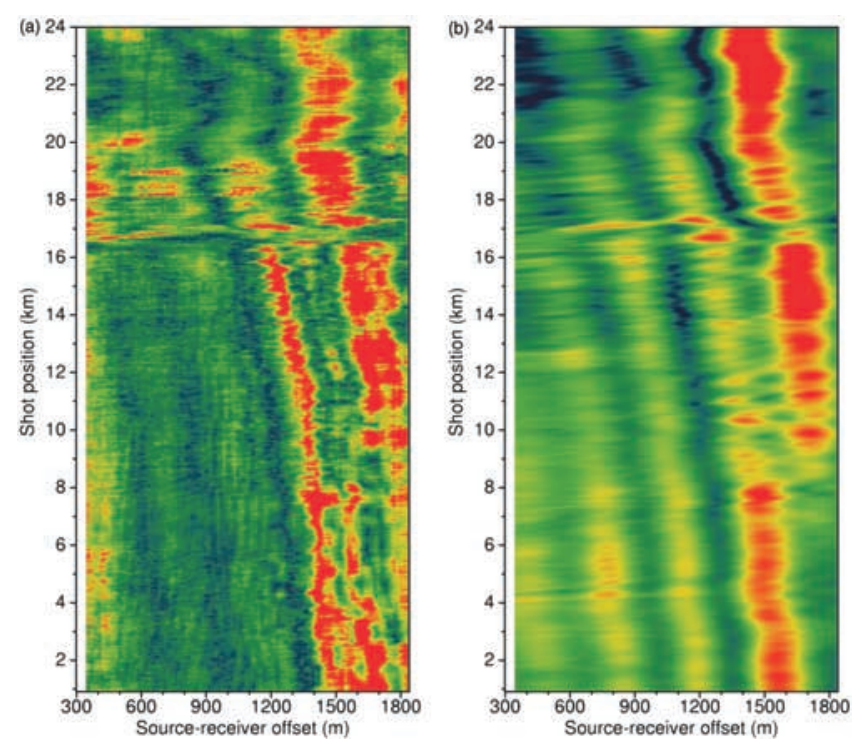

Figure 12. (a) A sample amplitude slice of real seismic data at frequency $9.3 \mathrm{~Hz}$. (b) The amplitude slice of synthetics at the same frequency, obtained from the waveform tomography result.

[40] In the inversion example shown in Figure 11, we use the scale factors $\beta(z)=1.0,1.14,1.4,1.7$, and 2.0 , for depth $z=0,350,800,1150,1500 \mathrm{~m}$, respectively. These are estimated using equation (15) with corresponding velocity $c(\mathrm{z})=1500,2000,2500,3000$ and $3300 \mathrm{~m} / \mathrm{s}$, respectively.

[41] Although the initial model generated by traveltime tomography generates a smooth boundary for the water bottom, waveform tomography produces a sharp geometry with a spatial variation close to that shown in seismic sections (Figure 9). From the waveform tomography image, we can see clearly a stratified structure under the water bottom. In the second layer, at the distance between 0 and $10 \mathrm{~km}$, there is a high-low-high vertical velocity variation immediately underneath the water bottom, between depth of 350 and $700 \mathrm{~m}$ at the left end of the profile, and a high-lowhigh-low vertical variation above the second interface. At the distance between 17 and $23 \mathrm{~km}$, there is also a lowhigh-low vertical velocity variation within the second layer.

[42] Most significantly, in the third layer, high-low-high velocity channels appear immediately underneath the second interface and cross the entire section. These thin-layer separations could not be generated by any conventional traveltime tomography. Beneath that, the velocity pattern in the depth between 1.2 and $1.6 \mathrm{~km}$ varies laterally between 0 and $5,5-17$, and $17-23 \mathrm{~km}$ in distance.

[43] Figure 12 compares a sample frequency slice (at $9.3 \mathrm{~Hz}$ ) of real data input to waveform tomography and synthetic data generated from the inversion result. The vertical axis is the shot position in the surface, and the horizontal axis is the source-receiver offset. The data displayed are the amplitudes of complex-valued samples in the frequency domain, and are normalized on the basis of their $r m s$ values. For the far-offset seismic traces, there are strong amplitudes for postcritical angle reflections, which play an important role in the inversion for the shallow part of model.
There is also a good fit at near-offset traces, which influence the high-wave number perturbations of the velocity field.

\section{Conclusions}

[44] Using reflection seismic data in waveform tomography, it is difficult to reconstruct the deep part of the model. A layer-stripping scheme can gradually improve the resolution of the image of the deep part. After inverting for the uppermost layer, the top portion of the data is not used in subsequent data fitting, and the inversion focuses on model updates in deeper parts of the model. A weighted-updating scheme can also be used to improve the deeper image, and the weight factor is set to be inversely proportional to the ray density which depends on depth. The latter scheme is more efficient in terms of computation time than the layerstripping scheme.

[45] When applying waveform tomography to real seismic data, raw shot records need be amplitude modified and phase adjusted, to make the data equivalent to those generated from a line source. As the real data lacks low-frequency information, reflection traveltime inversion may be used to provide a reliable initial model for the frequency domain waveform inversion. Waveform tomography uses a group of frequencies simultaneously in an iterative inversion, and proceeds from low to high frequencies. A real data example demonstrates that waveform tomography can generate a high-resolution image of subsurface velocities with detailed spatial variation, in contrast to image derived from conventional traveltime tomographic inversions.

[46] Acknowledgments. We are grateful to the sponsors of the Centre for Reservoir Geophysics, Imperial College London, for supporting this research.

\section{References}

Alford, R. M., K. R. Kelly, and D. M. Boore (1974), Accuracy of finitedifference modelling of the acoustic wave equation, Geophysics, 39 , 834-842, doi:10.1190/1.1440470.

Bleibinhaus, F., J. A. Hole, T. Ryberg, and G. S. Fuis (2007), Structure of the California Coast Ranges and San Andreas Fault at SAFOD from seismic waveform inversion and reflection imaging, J. Geophys. Res., 112, B06315, doi:10.1029/2006JB004611.

Brenders, A. J., and R. G. Pratt (2007a), Full waveform tomography for lithospheric imaging: Results from a blind test in a realistic crustal model, Geophys. J. Int., 168, 133-151, doi:10.1111/j.1365-246X.2006.03156.x.

Brenders, A. J., and R. G. Pratt (2007b), Efficient waveform tomography for lithospheric imaging: Implications for realistic, two-dimensional acquisition geometries and low-frequency data, Geophys. J. Int., 168, 152-170, doi:10.1111/j.1365-246X.2006.03096.x

Bunks, C., F. M. Saleck, S. Zaleski, and G. Chavent (1995), Multiscale seismic waveform inversion, Geophysics, 60, 1457-1473, doi:10.1190/ 1.1443880 .

Deregowski, S. M., and S. M. Brown (1983), A theory of acoustic diffractors applied to 2-D models, Geophys. Prospect., 31, 293-333, doi:10.1111/ j.1365-2478.1983.tb01055.x.

Helmberger, D. V., X. J. Song, and L. Zhu (2001), Crustal complexity from regional waveform tomography: Aftershocks of the 1992 Landers earthquake, California, J. Geophys. Res., 106(B1), 609-620, doi:10.1029/ 2000JB900231.

Hicks, G., and R. G. Pratt (2001), Reflection waveform inversion using local descent methods: Estimating attenuation and velocity over a gas-sand deposit, Geophysics, 66, 598-612, doi:10.1190/1.1444951.

Kelly, K. R., S. Treitel, and R. M. Alford (1976), Synthetic seismograms: A finite difference approach, Geophysics, 41, 2-27, doi:10.1190/ 1.1440605 .

Levander, A. R. (1988), Fourth-order finite-difference P-SV seismograms, Geophysics, 53, 1425-1436, doi:10.1190/1.1442422. 
Min, D. J., C. Shin, B. D. Kwon, and S. Chung (2000), Improved frequencydomain elastic wave modelling using weighted-averaging difference operators, Geophysics, 65, 884-895, doi:10.1190/1.1444785.

Mora, P. (1988), Elastic wave-field inversion of reflection and transmission data, Geophysics, 53, 750-759, doi:10.1190/1.1442510.

Operto, S., J. Virieux, J.-X. Dessa, and G. Pascal (2006), Crustal seismic imaging from multifold ocean bottom seismometer data by frequency domain full waveform tomography: Application to the eastern Nankai trough, J. Geophys. Res., 111, B09306, doi:10.1029/2005JB003835.

Pollitz, F. F., and J. P. Fletcher (2005), Waveform tomography of crustal structure in the south San Francisco Bay region, J. Geophys. Res., 110, B08308, doi:10.1029/2004JB003509.

Pratt, R. G. (1990), Frequency domain elastic wave modelling by finite differences: A tool for crosshole seismic imaging, Geophysics, 55, 626632, doi:10.1190/1.1442874.

Pratt, R. G. (1999), Seismic waveform inversion in the frequency domain, part 1: Theory and verification in a physical scale model, Geophysics, 64, 888-901, doi:10.1190/1.1444597.

Pratt, R. G., and R. M. Shipp (1999), Seismic waveform inversion in the frequency domain, part 2: Fault delineation in sediments using crosshole data, Geophysics, 64, 902-914, doi:10.1190/1.1444598.

Pratt, R. G., and M. H. Worthington (1990), Inverse theory applied to multisource cross-hole tomography. Part I: Acoustic wave-equation method, Geophys. Prospect., 38, 287-310, doi:10.1111/j.13652478.1990.tb01846.x

Pratt, R. G., Z.-M. Song, P. Williamson, and M. Warner (1996), Twodimensional velocity models from wide-angle seismic data by wavefield inversion, Geophys. J. Int., 124, 323-340, doi:10.1111/j.1365-246X. 1996.tb07023.x.

Pratt, R. G., C. Shin, and G. J. Hicks (1998), Gauss-Newton and full Newton methods in frequency-space seismic waveform inversion, Geophys. J. Int., 133, 341-362, doi:10.1046/j.1365-246X.1998.00498.x.

Pratt, R. G., F. Hou, K. Bauer, and M. H. Weber (2004), Waveform tomography images of velocity and inelastic attenuation from the Mallik 2002 crosshole seismic surveys, in Scientific Results From the Mallik 2002 Gas Hydrate Production Research Well Program, Mackenzie Delta Northwest Territories, Canada, edited by S. R. Dallimore and T. S. Collett, Bull. Geol. Surv. Can., vol. 585, 14 pp.

Priestley, K., E. Debayle, D. McKenzie, and S. Pilidou (2006), Upper mantle structure of eastern Asia from multimode surface waveform tomography, J. Geophys. Res., 111, B10304, doi:10.1029/2005JB004082.

Rao, Y., Y. Wang, and J. V. Morgan (2006), Crosshole seismic waveform tomography-II: Resolution analysis, Geophys. J. Int., 166, 1237-1248, doi:10.1111/j.1365-246X.2006.03031.x.

Ravaut, C., S. Operto, L. Improta, J. Virieux, A. Herrero, and P. Dell'Aversana (2004), Multiscale imaging of complex structures from multifold wideaperture seismic data by frequency-domain full-waveform tomography: Application to a thrust belt, Geophys. J. Int., 159, 1032-1056, doi:10.1111/j.1365-246X.2004.02442.x.

Shin, C., and D.-J. Min (2006), Waveform inversion using a logarithmic wavefield, Geophysics, 71, R31-R42, doi:10.1190/1.2194523.
Shin, C., S. Jang, and D.-J. Min (2001), Improved amplitude preservation for prestack depth migration by inverse scattering theory, Geophys. Prospect. 49, 592-606, doi:10.1046/j.1365-2478.2001.00279.x.

Sirgue, L., and R. G. Pratt (2004), Efficient waveform inversion and imaging: A strategy for selecting temporal frequencies and waveform inversion, Geophysics, 69, 231-248, doi:10.1190/1.1649391.

Snieder, R., M. Y. Xie, A. Pica, and A. Tarantola (1989), Retrieving both the impedance contrast and background velocity: A global strategy for the seismic reflection problem, Geophysics, 54, 991-1000, doi:10.1190/ 1.1442742 .

Song, Z.-M., and P. R. Williamson (1995), Frequency-domain acousticwave modeling and inversion of crosshole data: Part I-2.5-D modeling method, Geophysics, 60, 784-795, doi:10.1190/1.1443817.

Song, Z.-M., P. R. Williamson, and R. G. Pratt (1995), Frequency-domain acoustic-wave modeling and inversion of crosshole data: Part IIInversion method, synthetic experiments and real-data results, Geophysics, 60, 796-809, doi:10.1190/1.1443818.

Štekl, I., and R. G. Pratt (1998), Accurate viscoelastic modeling by frequency-domain finite differences using rotated operators, Geophysics, $63,1779-1794$, doi:10.1190/1.1444472.

Tarantola, A. (1984), Inversion of seismic reflection data in the acoustic approximation, Geophysics, 49, 1259-1266, doi:10.1190/1.1441754.

Tarantola, A. (1987), Inverse Problem Theory: Methods for Data Fitting and Model Parameter Estimation, Elsevier, Amsterdam.

Tarantola, A. (2005), Inverse Problem Theory and Methods for Model Parameter Estimation, Soc. for Ind. and Appl. Math., Philadelphia, Pa.

Virieux, J. (1986), $P-S V$ wave-propagation in heterogeneous media: Velocitystress finite-difference method, Geophysics, 51, 889-901, doi:10.1190/ 1.1442147.

Wang, Y. (2003), Seismic Amplitude Inversion in Reflection Tomography, Elsevier Sci., Amsterdam.

Wang, Y. (2004), Multiple prediction through inversion: A fully data-driven concept for surface-related multiple attenuation, Geophysics, 69, $547-$ 553, doi:10.1190/1.1707074.

Wang, Y. (2007), Multiple prediction through inversion: Theoretical advancements and real data application, Geophysics, 72, V33-V39, doi:10.1190/1.2408379.

Wang, Y, and G. A. Houseman (1995), Tomographic inversion of reflection seismic amplitude data for velocity variation, Geophys. J. Int., 123, 355372, doi:10.1111/j.1365-246X.1995.tb06859.x.

Wang, Y., and Y. Rao (2006), Crosshole seismic waveform tomographyI: Strategy for real data application, Geophys. J. Int., 166, 1224-1236, doi:10.1111/j.1365-246X.2006.03030.x.

Wu, R., and M. N. Toksöz (1987), Diffraction tomography and multisource holography applied to seismic imaging, Geophysics, 52, 11-25, doi:10.1190/1.1442237.

Zhou, B., and S. A. Greenhalgh (2003), Crosshole seismic inversion with normalized full-waveform amplitude data, Geophysics, 68, 1320-1330, doi:10.1190/1.1598125.

Y. Rao and Y. Wang, Centre for Reservoir Geophysics, Department of Earth Science and Engineering, Imperial College London, South Kensington, London SW7 2BP, UK. (yanghua.wang@imperial.ac.uk) 